\title{
Extensions to Common Laplace and Fourier Transforms
}

\author{
Levent Onural, Senior Member, IEEE, M. Fatih Erden, and Haldun M. Ozaktas
}

\begin{abstract}
The extended versions of common Laplace and Fourier transforms are given. This is achieved by defining a new function $f_{e}(p), p \in C$ related to the function to be transformed $f(t), t \in R$. Then $f_{e}(p)$ is transformed by an integral whose path is defined on an inclined line on the complex plane. The slope of the path is the parameter of the extended definitions which reduce to common transforms with zero slope. Inverse transforms of the extended versions are also defined. These proposed definitions, when applied to filtering in complex ordered fractional Fourier stages, significantly reduce the required computation.
\end{abstract}

Index Terms-Filtering, Fourier transform, fractional Fourier transform, Laplace transform.

\section{BASIC DEFINITIONS AND INTERPRETATIONS}

$\mathbf{I}$ T IS possible to extend the common definitions of Laplace and Fourier transforms; the benefits are numerous. An extension is presented in this paper, together with its application to a specific problem. The solution of the problem is significantly simplified by the presented extension.

We adopt the following Fourier and two-sided Laplace transforms:

$$
\begin{aligned}
& \left.\mathcal{F}\{f\}\right|_{t, \omega}=\int_{-\infty}^{\infty} f(t) e^{-j \omega t} d t \quad t, w \in \mathbf{R} \\
& \left.\mathcal{L}\{f\}\right|_{t, s}=\int_{-\infty}^{\infty} f(t) e^{-s t} d t \quad t \in \mathbf{R}, s \in \mathbf{C} .
\end{aligned}
$$

The inversion formulas can be written, accordingly.

Let us define two complex planes, $p$-plane and $s$-plane, as $p=t+j \tau$ and $s=\sigma+j \omega$. Furthermore, let us define an $\alpha$-axis on $p$-plane as a straight line that passes through the origin making an angle $\alpha$ with the real axis as shown in Fig. 1. A $\theta$-axis is defined similarly on the $s$-plane. These lines are denoted as $L_{\alpha}$ and $L_{\theta}$, respectively.

Suppose that a function $f: \mathbf{R} \rightarrow \mathbf{C}$ is given; i.e., in $f(t) t$ is real valued but $f(t)$ may take complex values. Let us define another function $f_{e}: \mathbf{C} \rightarrow \mathbf{C}$, which is related to $f$ as follows:

$$
f_{e}(p)=\left.f(t)\right|_{t \rightarrow p}, \quad t \in \mathbf{R}, p \in \mathbf{C} .
$$

The real and imaginary parts of $f_{e}(p)$, related to $f(t)=e^{-t}$, are shown in Fig. 2.

Here is the extended version of the Fourier transform, $\mathcal{F}_{e}$, of $f(t)$ (which can also be viewed as the extended version of

Manuscript received November 5, 1996. The associate editor coordinating the review of this manuscript and approving it for publication was Prof. R. Mersereau.

The authors are with the Department of Electrical Engineering, Bilkent University, TR-06533 Bilkent, Ankara, Turkey (e-mail: onural@ ee.bilkent.edu.tr).

Publisher Item Identifier S 1070-9908(97)08213-8.

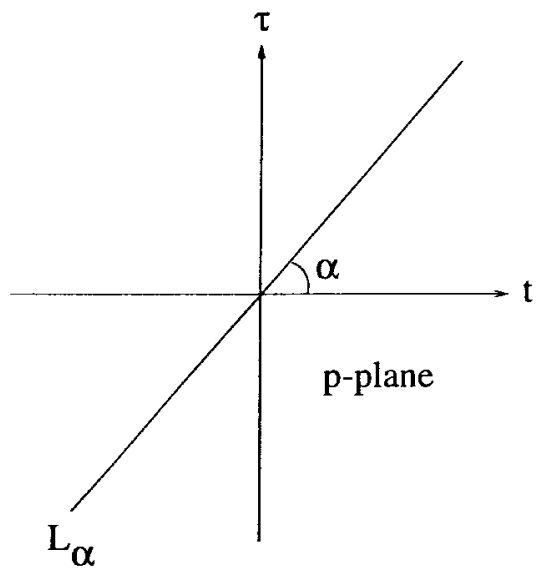

(a)

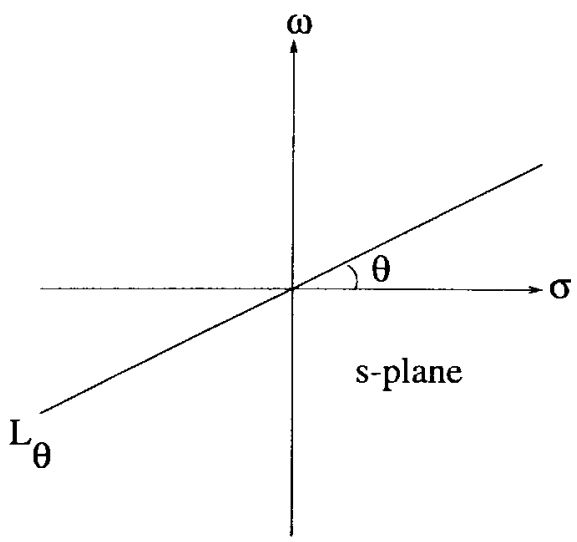

(b)

Fig. 1. Lines $L_{\alpha}$ and $L_{\theta}$ with slopes $\alpha$ and $\theta$ are shown on two separate complex planes.

the Laplace transform):

$$
\left.\mathcal{F}_{e}\{f(t)\}\right|_{p, s}=\mathcal{F}_{e}(s)=\int_{L_{\alpha}} f_{e}(p) e^{-s p} d p
$$

The inverse transform then becomes

$$
f(t)=\left.f_{e}(p)\right|_{p \rightarrow t}=\left.\frac{1}{2 \pi} \int_{L_{\theta}} \mathcal{F}_{e}(s) e^{s p} d s\right|_{p \rightarrow t}
$$

if $\theta+\alpha=\pi / 2$.

The extension as described above can be interpreted in various ways. Here is one of them: What is first achieved by replacing the real $t$ by a complex $p$ in a given $f(t)$ is a conversion of a one-dimensional (1-D) real variable function to a two-dimensional (2-D) real variable function (function of a complex variable). The line $L_{\alpha}$ gives a 1-D profile of 

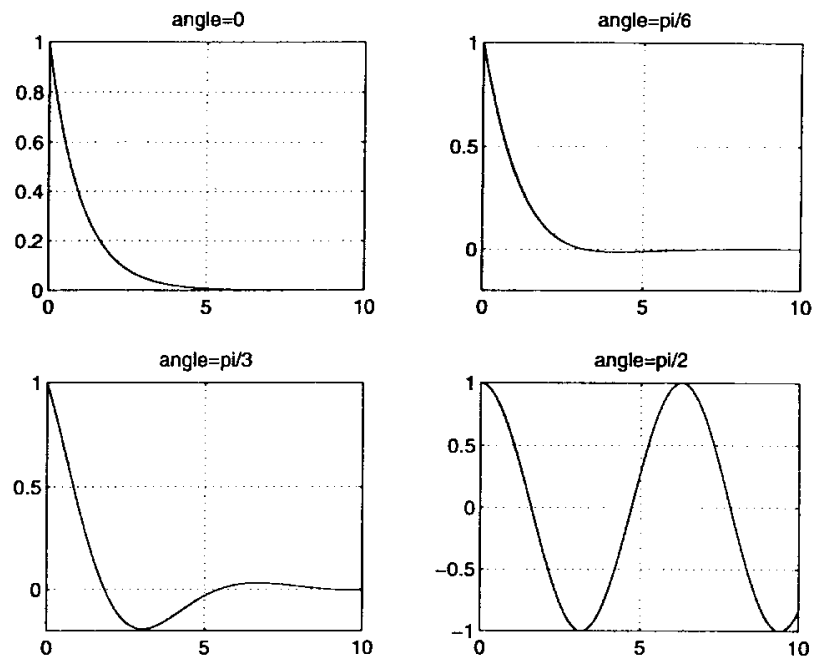

(a)
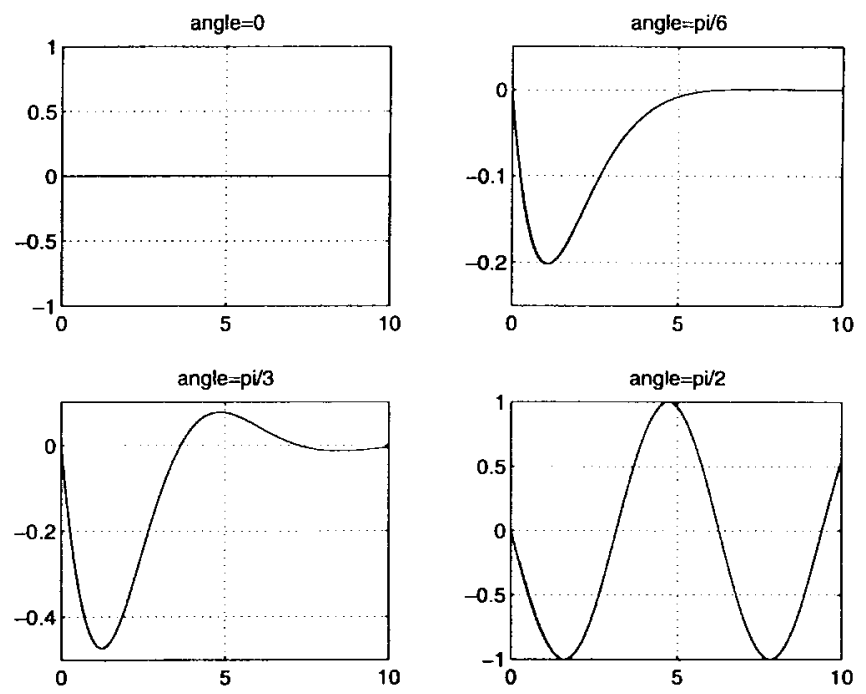

(b)

Fig. 2. Real and imaginary parts of $f_{e}(p)$ when $f(t)=e^{-t}$ in (3). (a) Real part. (b) Imaginary part.

this 2-D function. Knowing any such profile (provided that $\alpha$ is known, too) the complete 2-D function is also known. However, now we have a choice of an $\alpha$ (in the common definition, the $\alpha$ is always zero), which would give a 1-D function that is more suitable for Fourier operations for a given purpose. The $\mathcal{F}_{e}$ is also a 2-D real variable function; the 1-D profile over $L_{\theta}$ becomes the common 1-D Fourier transform of the 1-D function over $L_{\alpha}$ provided that $\theta+\alpha=\pi / 2$. This relationship may be exploited in various applications. Another interpretation can be made in terms of the integration paths on the complex planes: The common transform and its inversion are evaluated by integrals over $t$-axis (pure real) and $\omega$-axis (pure imaginary), respectively. Now, the transform integral path is rotated by an angle $\alpha$, together with a conversion of a function of a real variable to a function of a complex variable. As a consequence, the path of the inverse transform on the complex-plane is also rotated by an angle $\pi / 2-\alpha$.

Note that sampling of the extended Fourier and inverse transforms as described in (4) and (5) is straightforward.

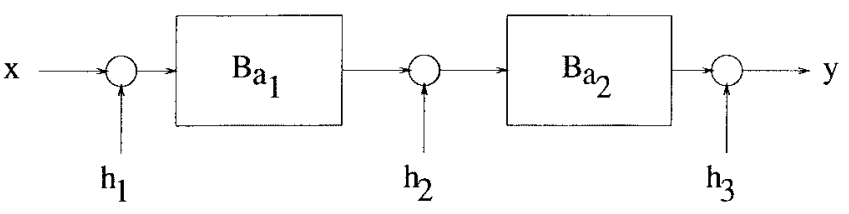

Fig. 3. This configuration performs filtering in complex order fractional Fourier domains. $B_{a_{1}}$ and $B_{a_{2}}$ are the kernels of the fractional Fourier transform of (6) corresponding to complex fractions $a_{1}$ and $a_{2}$, respectively. The multipliers $h_{1}(\cdot), h_{2}(\cdot), h_{3}(\cdot)$, and the complex orders $a_{1}, a_{2}$ are known. We obtain the filtered output $y(\cdot)$ for any input $x(\cdot)$.

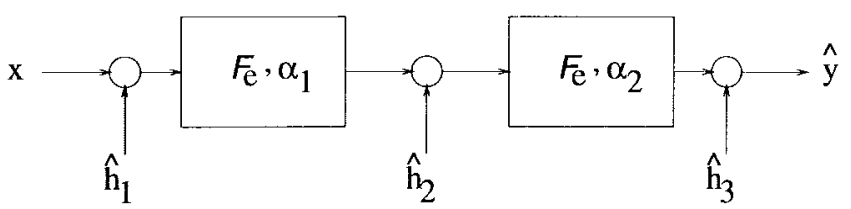

Fig. 4. Equivalent system of the configuration shown in Fig. 3. $\mathcal{F}$ together with $\alpha_{1}$ and $\alpha_{2}$ indicates the extended Fourier transform of (4) with transform integral paths $L_{\alpha_{1}}$ and $L_{\alpha_{2}}$, respectively. The multipliers $\hat{h}_{1}(\cdot), \hat{h}_{2}(\cdot), \hat{h}_{3}(\cdot)$, the output $\hat{y}(\cdot)$, and the angles $\alpha_{1}$ and $\alpha_{2}$ of the extended Fourier transform stages are obtained from (8)-(12).

Therefore, corresponding discrete transform can be obtained. This is essential for efficient numerical computations.

\section{AN APPLICATION}

The $a$ th-order fractional Fourier transform $\left(\mathbf{F}^{a} \hat{q}\right)(t)$ of the function $\hat{q}(t)$ is defined for $0<|a|<2$ as

$$
\begin{aligned}
\left(\mathbf{F}^{a} \hat{q}\right)(t) & \triangleq \int_{-\infty}^{\infty} B_{a}\left(t, t^{\prime}\right) \hat{q}\left(t^{\prime}\right) d t^{\prime} \\
B_{a}\left(t, t^{\prime}\right) & \triangleq \mathcal{K} \exp \left[j \pi\left(\gamma t^{2}-2 \beta t t^{\prime}+\gamma t^{\prime 2}\right)\right]
\end{aligned}
$$

where $\mathcal{K}, \gamma$ and $\beta$ are functions of the order $a$, and can be expressed as

$\mathcal{K} \triangleq \frac{\exp [-j(\pi \hat{\phi} / 4-\phi / 2)]}{|\sin \phi|^{1 / 2}}, \quad \gamma \triangleq \cot \phi, \beta \triangleq \csc \phi$

with $\phi=a \pi / 2$ and $\hat{\phi}=\operatorname{sgn}(\sin \phi)$. The kernel is defined separately for $a=0$ and $a= \pm 2$ as $B_{0}\left(t, t^{\prime}\right) \triangleq \delta\left(t-t^{\prime}\right)$ and $B_{ \pm 2}\left(t, t^{\prime}\right) \triangleq \delta\left(t+t^{\prime}\right)$, respectively [1]. The definition is easily extended outside the interval $[-2,2]$ by noting that $\mathcal{F}^{4 k+a} \hat{q}=\mathcal{F}^{a} \hat{q}$ for any integer $k$. Both $t$ and $t^{\prime}$ are interpreted as dimensionless variables. The properties of the fractional Fourier transform may be found in [1]-[6].

Note that $a$ in (6) is taken as a real variable in most of the cases. However, it can also be a complex variable [7]. Let us consider the filtering problem shown in Fig. 3, which represents a physical setup when the signals are 2-D. For simplicity, we analyze the 1-D version here. In this figure, we have two complex order fractional Fourier transform stages sandwiched between three multipliers. The multipliers $h_{1}(\cdot)$, $h_{2}(\cdot), h_{3}(\cdot)$, and the complex orders $a_{1}, a_{2}$ are known. We want to obtain the output $y(\cdot)$ for any input $x(\cdot)$. After straightforward algebra, we obtain the equivalent system as shown in Fig. 4. In this figure, the multipliers $\hat{h}_{1}(\cdot), \hat{h}_{2}(\cdot)$, $\hat{h}_{3}(\cdot)$, the output $\hat{y}(\cdot)$, and the angles $\alpha_{1}$ and $\alpha_{2}$ of the extended 
Fourier transform stages are related to $h_{1}(\cdot), h_{2}(\cdot), h_{3}(\cdot), a_{1}$, and $a_{2}$ as

$$
\begin{aligned}
\hat{h}_{1}(t) & =\mathcal{K}_{1} h_{1}(t) \exp \left(j \pi \gamma_{1} t^{2}\right), \\
\hat{h}_{2}(t) & =\frac{1}{\left|\beta_{1}\right|} h_{2}\left(\frac{t}{\left|\beta_{1}\right|}\right) \exp \left[j \pi \frac{\left(\gamma_{1}+\gamma_{2}\right) t^{2}}{\left|\beta_{1}\right|^{2}}\right] \\
\hat{h}_{3}(t) & =h_{3}\left(\frac{\left|\beta_{1}\right|}{\left|\beta_{2}\right|} t\right) \exp \left(j \pi \gamma_{2} \frac{\left|\beta_{1}\right|^{2} t^{2}}{\left|\beta_{2}\right|^{2}}\right) \\
\hat{y}(t) & =y\left(\frac{\left|\beta_{1}\right|}{\left|\beta_{2}\right|} t\right) \\
& \alpha_{1}=\arg \left(\beta_{1}\right), \quad \alpha_{2}=\arg \left(\beta_{2}\right) .
\end{aligned}
$$

In these equations, $\mathcal{K}_{1}, \gamma_{1}$, and $\beta_{1}$ are associated with $a_{1}$; and $\mathcal{K}_{2}, \gamma_{2}$, and $\beta_{2}$ are associated with $a_{2}$ through (6). Thus, the problem of filtering in complex order fractional Fourier domains can also be expressed in terms of the proposed extended Fourier transform definition of (4).

We do not have a direct fast computational algorithm for the complex order fractional Fourier transform. However, since Figs. 3 and 4 are equivalent, the computation of filtering in complex order fractional Fourier domains (Fig. 3) can now be carried out by the discrete version of the extended Fourier transform (Fig. 4). Thus, a fast computation is now achieved.

\section{CONCLUSION}

In this letter, it is shown that the extended definitions of the common Laplace and Fourier transforms provide a useful framework for some applications. These extended definitions are more general than the conventional counterparts and, thus, may pave the road for many new applications.

\section{REFERENCES}

[1] A. C. McBride and F. H. Kerr, "On Namias's fractional Fourier transform,” IMA J. Appl. Math., vol. 39, pp. 159-175, 1987.

[2] D. Mendlovic and H. M. Ozaktas, "Fractional Fourier transforms and their optical implementation, I," J. Opt. Soc. Amer. A., vol. 10, pp. 1875-1881, 1993.

[3] H. M. Ozaktas and D. Mendlovic, "Fractional Fourier transforms and their optical implementation, II," J. Opt. Soc. Amer. A., vol. 10, pp. 2522-2531, 1993.

[4] A. W. Lohmann, "Image rotation, Wigner rotation, and the fractional order Fourier transform," J. Opt. Soc. Amer. A., vol. 10, pp. 2181-2186, 1993.

[5] H. M. Ozaktas, B. Barshan, D. Mendlovic, and L. Onural, "Convolution, filtering, and multiplexing in fractional Fourier domains and their relation to chirp and wavelet transforms," J. Opt. Soc. Amer. A., vol. 11, pp. 547-559, 1994.

[6] L. B. Almeida, "The fractional Fourier transform and time-frequency representations," IEEE Trans. Signal Processing, vol. 42, pp. 3084-3091, 1994.

[7] C. Shih, "Optical interpretation of a complex-order Fourier transform," Opt. Lett., vol. 20, pp. 1178-1180, 1995. 